\title{
Plant Species Classification using a 3D LIDAR Sensor and Machine Learning
}

\author{
Ulrich Weiss and Peter Biber \\ Corporate Sector Research and Advance Engineering, \\ Robert Bosch GmbH \\ Schwieberdingen, Germany \\ \{ulrich.weiss,peter.biber\}@de.bosch.com
}

\author{
Stefan Laible, Karsten Bohlmann and Andreas Zell \\ Wilhelm-Schickard-Institute for Computer Science (WSI), \\ University of Tuebingen, \\ Tuebingen, Germany \\ $\{$ stefan.laible,karsten.bohlmann,andreas.zell $\} @$ uni-tuebingen.de
}

\begin{abstract}
In the domain of agricultural robotics, one major application is crop scouting, e.g., for the task of weed control. For this task a key enabler is a robust detection and classification of the plant and species. Automatically distinguishing between plant species is a challenging task, because some species look very similar. It is also difficult to translate the symbolic high level description of the appearances and the differences between the plants used by humans, into a formal, computer understandable form. Also it is not possible to reliably detect structures, like leaves and branches in 3D data provided by our sensor. One approach to solve this problem is to learn how to classify the species by using a set of example plants and machine learning methods.

In this paper we are introducing a method for distinguishing plant species using a 3D LIDAR sensor and supervised learning. For that we have developed a set of size and rotation invariant features and evaluated experimentally which are the most descriptive ones. Besides these features we have also compared different learning methods using the toolbox Weka. It turned out that the best methods for our application are simple logistic regression functions, support vector machines and neural networks. In our experiments we used six different plant species, typically available at common nurseries, and about 20 examples of each species. In the laboratory we were able to identify over $98 \%$ of these plants correctly.
\end{abstract}

Keywords-plant classification; supervised learning; 3D laser sensor; agricultural robotics

\section{INTRODUCTION}

In the domain of agriculture the concept of precision agriculture is increasingly being recognized and gets more and more important, e.g., for financial, time saving and environmental protection reasons. Precision agriculture is about applying the right treatment, at the right place and at the right time [1]. To be able to make decisions about such treatments, it is necessary to collect, store and process crop field data at a subfield level. For that, an important prerequisite is to reliably detect and classify the plants on the field.

The individual plant inspection and treatment is a very labor-intensive practice and brings a great need for automation, for instance by using autonomous vehicles. In the future, such vehicles are needed for soil sampling, crop scouting, and real-time data collection [2]. Small robots are well suited for these tasks of individual plant care, such as selective crop harvesting and individual crop rating as

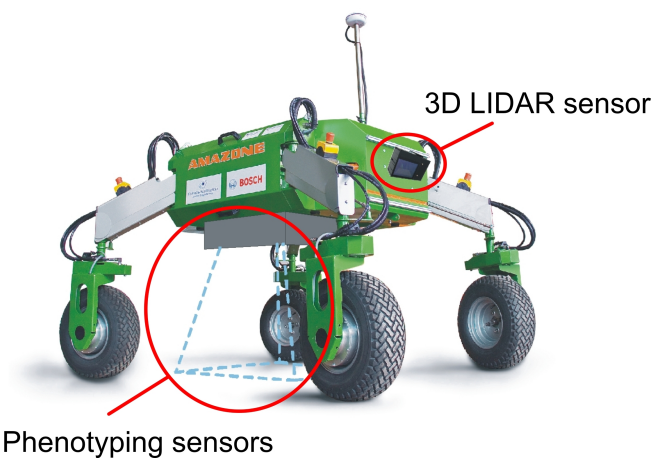

(a) BoniRob

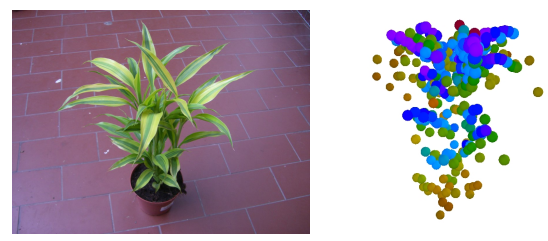

(b) Dracaena sanderina

Figure 1. (a) shows the BoniRob platform. (b) shows an example plant and the corresponding point cloud. The point cloud is colored according to the reflectance values using the HSV color space.

well as precise spraying, fertilization and selective weed control, with the effects of reducing costs and consumption of fertilizers and pesticides.

The work presented here is a part of the publicly funded project BoniRob [3], a cooperation of academic and industrial partners, with the goal to develop an autonomous mobile agricultural robot (see Figure 1(a)). The key objective of BoniRob is the autonomous repeating phenotyping of individual plants at different days, to track the growth stage and the condition of the plants. For that it is necessary to detect and map single plants. To map only the crops, the robot has to be able to distinguish between different plant species.

In a previous publication [4] we have already shown, that we are able to detect and map single plants. We have proposed an algorithm performing individual plant detection and mapping using a 3D laser sensor on a mobile robot. Our algorithm is based on an approach detecting the ground to 
segment the point cloud into soil and other objects. In the remaining point cloud the algorithm searches for clusters representing individual plants using a model of a crop row. We have shown that this task can be reliably carried out using the 3D sensor with 59x29 rays. The 3D laser sensor has also shown that it is robust against illumination and atmospheric conditions, which enables the robot to operate reliably at any weather condition even 24 hours a day.

For a species based mapping the next step is to classify the plants. In this paper we present an approach for distinguishing different plant species using the 3D laser sensor and machine learning methods. We are using the above mentioned algorithm to detect the plants and use the resulting clusters as input for the classification.

To associate a cluster with a plant species is a challenging task. Some species look similar and in the sparsely populated point cloud (see Figure 1(b)) it is not possible to reliably recognize structures like leaves and branches. Furthermore, it is difficult to translate the high level description used by humans to identify plant species into a formal, computer understandable description. Botanists are using field guides to identify plants, but they are getting better and better the more they observe samples of plant species-they are learning the classification process. Because of these reasons we are using supervised offline learning to classify the species by using example plants. A further advantage of the classification using machine learning methods is the fast online processing.

To classify the species we have developed a set of simple, easy and fast to calculate features to describe the plant species. These features have been developed according to our requirements: The classification should run in realtime; we are also only using size independent and rotation invariant features. The size independence is meaningful if the plants looks similar at different growth stages. The rotation invariance is important, because the plants should be classified from different points of view. Our features can be divided into two groups-in reflectance value based and in geometrical features. Mainly we are using statistical values describing the distribution of the data, like minimum, maximum, standard deviation and histograms.

To learn and to compare these features, we have decided to use the machine learning toolbox Weka [5]. We compared the different groups of learners as well as the individual learners, to find the best methods for our application. To create a database for our experiments we have chosen six different plant species, typically available at common nurseries, and about 20 examples of each species. In the laboratory we were able to identify over $98 \%$ of these plants correctly.

This paper is organized as follows. In section II related work, dealing with plant and object detection using different sensor systems, is discussed. The plant classification algorithm and the developed feature set are presented in section III. Section IV shows the experimental results. The paper closes with conclusions in section V.

\section{RELATED WORK}

In this section the related work dealing with crop/weeddiscrimination, plant detection and plant classification, using different sensor systems, like vision, stereo-vision, laser sensors and ultrasonic sensors are discussed. Further, works dealing with 3D object recognition and feature-based learning of objects and structures are considered.

Tang et al. have introduced and compared two techniques for corn plant and population sensing using stereo vision [6]. For corn plant identification they are using leaf detection algorithms. To detect leafs in 3D images a high resolution and complex algorithms are required. One big disadvantage using vision or stereo vision is the influence by changing lighting conditions, which are not uncommon in outdoor applications like agriculture. Laser range finders are much more reliable in such situations. Up to now, laser sensors based applications are rare in this domain.

A work dealing with individual plant detection and mapping using a 3D laser sensor was proposed in [4]. We used a 3D laser sensor and a highly precise RTK-GPS on a robot, detecting the corn plants and determining their positions to build-up crop maps of row cultivations. Even in dense rows our approach achieved about $80 \%$ of correctly detected plants, but we did not discriminate between crop and weed.

Paap et al. [7] used a spectral reflectance sensor for ground-based plant detection and weed discrimination. They used a laser sensor with different near infrared wavelength and measured the slope between these wavelengths to determine the so called Normalized Differenced Vegetation Index (NDVI) to discriminate between soil, crop and weed.

A method to classify plants using ultrasonic signals accessible to bats was proposed in [8]. They are using spectrograms of echoes of plants and calculate features learned using a support vector machine. Works using such biosonar sensors for natural landmark classification were also proposed in [9] and [10]. Another method for plant classification was proposed by Wu et al. [11]. They are using a computer vision leaf recognition algorithm to determine features describing the leaf's shape and size, and a probabilistic neural network (PNN) to learn the relation. For their experiments they have used 32 kinds of plants and achieved a detection rate of about $90 \%$.

Besides the plant classification, there are many publications dealing with 3D object recognition and classification. In [12] Böhm et al. are using CAD models for curvature based range image classification. They extract features from surface curvatures using dense 3D images, and classify the objects using minimum distance matching. Hetzel et al. developed a method for 3D object recognition from range images using local multidimensional feature histograms [13]. They only used features which are easy to 


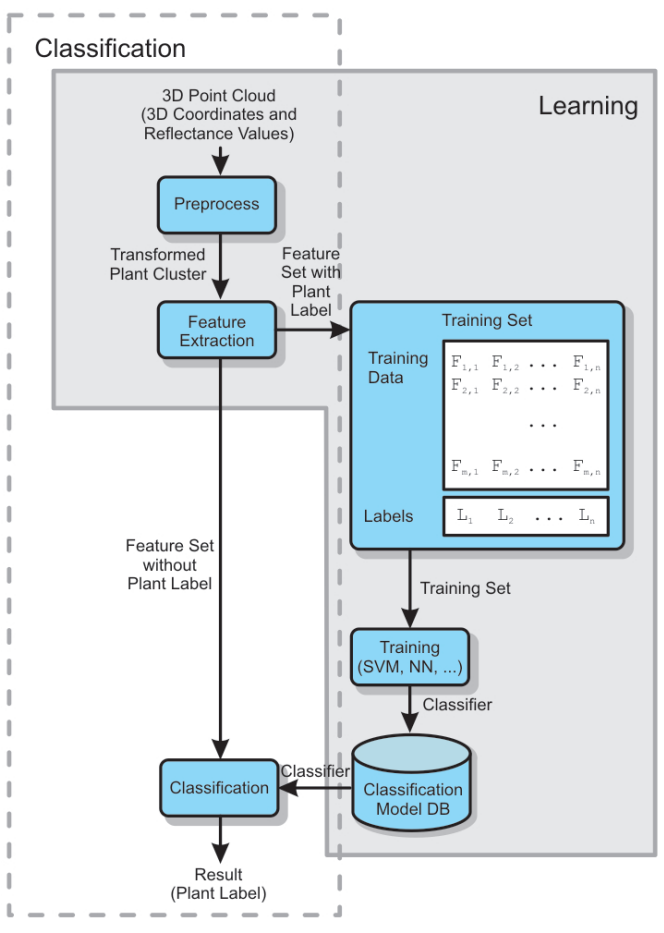

Figure 2. Algorithm overview: The grey box marks the learning and the dashed box the classification process.

calculate and they did not need image segmentation for their recognition algorithm. They were using histogram matching and achieved a detection rate of about $90 \%$.

Ghobadi et al. compared time-of-flight PMD cameras and stereo vision systems for detecting and classifying simple moving 3D objects using a multiclass Support Vector Machine. They implemented and compared two methods of feature generation. One automatically generates features using a Principal Component Analysis (PCA), the second uses human defined features. With the second approach and a time-of-flight camera they got detection rates of almost $100 \%$.

Indeed, there are some works dealing with plant detection and classification, but they are not feasible for our application. First, their sensors are not well suited for the agricultural robotics application, and second, our method has to run in real-time. The 3D laser sensor, used by us, has also many advantages in this environment and the use of simple and easy to calculate features is also advantageous.

\section{Plant Classification}

In this section an overview of the classification and learning algorithm is given. Further, the preprocessing of the data and the features will be described. This section closes with the selection of the learning methods.

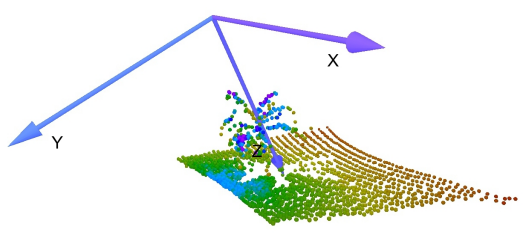

(a) Laser coordinate system

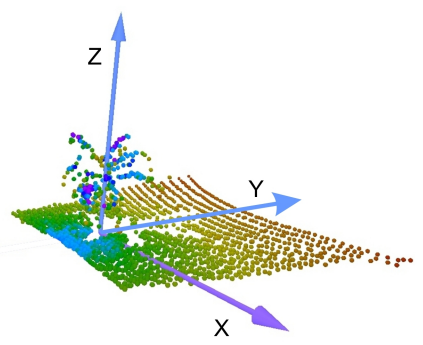

(b) Plant coordinate system

Figure 3. Transformation into plant coordinate system

\section{A. Algorithm Overview}

Figure 2 shows an overview of the classification and learning process. The input data are 3D point clouds with range and reflectance values from a 3D laser sensor. First some preprocessing techniques are applied to provide the point cloud for further processing. Then relevant features are derived. If the species of the sensed plant is known, the extracted features are added as new data to the training set, otherwise the features are used as input for the classification process. For the training dataset different classifiers are trained and stored in the classification model database. The learned classifier is applied to the features of the unlabeled $3 \mathrm{D}$ data and the label of the object is returned.

\section{B. Preprocessing}

The preprocessing consists of three steps. The first step is to detect the ground plane using a RANSAC algorithm and eliminate the points belonging to this ground plane. In the next step the remaining point cloud is clustered and individual plants are detected. In the third step we determine an individual coordinate system for each plant. The $\mathrm{x}$ - and $\mathrm{y}-$ axis of these coordinate systems are placed on the ground, the z-axis points upwards and is located at the center of the plant (see Figure 3). Finally, the points are transformed into the according plant coordinate system. This individual plant detection algorithm was already proposed in [4]. We showed that we are able to separate individual plants in row cultivations. What is missing is to distinguish between different plant species, e.g., between corn and weed.

\section{Feature Extraction}

Our 3D laser sensor provides two types of data: reflectance values and 3D coordinates. Using these data, we 
developed a set of features which describes the appearance of the plant species, with the criteria of size and rotation invariance. The features must be simple and also be fast to calculate, because our sensor has a low resolution and the classification should run in real-time. Our feature set can be divided into two main groups: Reflectance based and geometrical features. The geometrical features can also be divided into features considering the $3 \mathrm{D}$ distribution, the distribution into the paraxial planes according to the plant coordinate system, and the neighborhood of the points. In this section the 83 developed features are described.

1) Reflectance Features: Our experiments have shown that different plants species have broad variations with respect to the reflectance values. The reflectance value depend on the reflectance characteristic of the sensed object, but also on the angle of impact. Despite the last property the reflectance value is very useful for plant classification. Although the angle of impact can not be measured, the reflectance value encodes information about the leaf size and shape.

To encode the reflectance properties of the plants we have developed a set of statistical features. For each measuring point a reflectance value between 0 and 1 is determined.

- Feature 1: Minimum reflectance value

- Feature 2: Maximum reflectance value

- Feature 3: Range of the reflectance values

- Feature 4: Mean of the reflectance values

- Feature 5: Median of the reflectance values

- Feature 6: Standard deviation of the reflectance values

- Feature 7: Variance of the reflectance values

- Feature 8-16: Histogram of the reflectance values

The optimal number of bins for a histogram can be calculated using the rule of Sturgess $\left(\mid\right.$ bins $\left.\mid=\left\lceil\log _{2} n+1\right)\right\rceil$ ). In our application $\mid$ bins $\mid$ depends on the average number of points per plant. This number was experimentally determined and is about 243 , which leads to $\mid$ bins $\mid=9$. This value is also used for the geometrical feature histograms.

2) Geometrical Features: Besides the reflectance values the laser sensor provides range data which can be transformed into 3D coordinates. Using these coordinates we can calculate values characterizing the shape, dimension and the structure of the plant.

Let $P=\left\{\left(x_{1}, y_{1}, z_{1}\right),\left(x_{2}, y_{2}, z_{2}\right), \ldots,\left(x_{n}, y_{n}, z_{n}\right)\right\}$ be the 3D point cloud of a plant with respect to the plant coordinate system, with $n=|P|$ the number of points, $h=\max \left(\left\{y_{1}, y_{2}, \ldots y_{n}\right\}\right)$ the height of the plant, and $w=\max \left(\left\{\sqrt{x^{2}+z^{2}}:(x, y, z) \in P\right\}\right)$ the width of the plant. $n, h$ and $w$ are not size invariant and should not be used as features if different growing stages of the plants are expected. But by using these values, size invariant features like the ratio and the density of the point cloud can be determined.

- Feature 17: Ratio of height to width

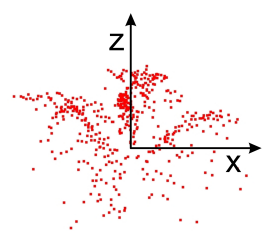

(a) Front-view $P_{x, z}$

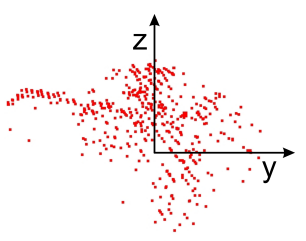

(b) Side-view $P_{y, z}$

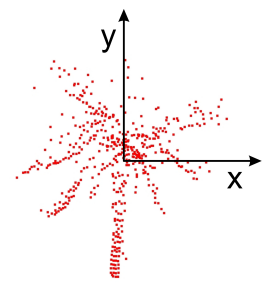

(c) Top-view $P_{x, z}$

Figure 4. The images show the 2D projection on front-, side- and top-view. The projections are created using the point cloud of a Guzmania Bromelia plant (see Figure 5(c)).

- Feature 18: Density with respect to bounding cylinder

$$
\rho=\frac{n}{\pi \cdot w^{2} \cdot h}
$$

Further 3D geometrical features depends on the distribution of the point cloud with respect to the cloud center $C$. For that, let

$$
D=\left\{\left|C-p_{1}\right|,\left|C-p_{2}\right|, \ldots\left|C-p_{n}\right|\right\}, \text { with } p_{i} \in P .
$$

By scaling $D$ with $\frac{1}{\max (D)}$ the distances can be transformed into the size independent description:

$$
\bar{D}=\left\{\frac{d_{1}}{d_{\max }}, \frac{d_{2}}{d_{\max }}, \ldots, \frac{d_{n}}{d_{\max }}\right\}, \text { with } d_{i} \in D .
$$

Using $\bar{D}$, similar to the reflectance based features, the following statistical features can be calculated.

- Feature 19: Mean of the distances from $C$

- Feature 20: Median of the distances from $C$

- Feature 21: Variance of the distances from $C$

- Feature 22: Standard dev. of the distances from $C$

- Feature 23-31: Histogram of the distances from $C$

These features are describing the distribution inside the point cloud.

Besides the 3D coordinates we have developed 2D based geometrical features. For that, the projections on the paraxial planes of the plant coordinate system are used. Figure 4 shows these front-, side- and top-view-projections. Despite the low resolution, these projections show characteristic structures, e.g. leaves. Due to the orientation of the sensor according to the plant the front- and side-view are not similar. 
Although the elements of these sets are two-dimensional, we can use the $2 \mathrm{D}$ distances from the center $C$ and get features analogical to the 3D geometrical features, respectively for each projection. Again the distances are normalized. These features are:

- Feature 32: Mean of the distances in $P_{y, z}$

- Feature 33: Median of the distances in $P_{y, z}$

- Feature 34: Variance of the distances in $P_{y, z}$

- Feature 35: Standard dev. of the distances in $P_{y, z}$

- Feature 36-44: Histogram of the distances in $P_{y, z}$

- Feature 45: Mean of the 2D distances in $P_{x, z}$

- Feature 46: Median of the 2D distances in $P_{x, z}$

- Feature 47: Variance of the 2D distances in $P_{x, z}$

- Feature 48: Standard dev. of the 2D distances in $P_{x, z}$

- Feature 49-57: Histogram of the $2 \mathrm{D}$ distances in $P_{x, z}$

- Feature 58: Mean of the 2D distances in $P_{x, y}$

- Feature 59: Median of the 2D distances in $P_{x, y}$

- Feature 60: Variance of the 2D distances in $P_{x, y}$

- Feature 61: Standard dev. of the 2D distances in $P_{x, y}$

- Feature 62-70: Histogram of the 2D distances in $P_{x, y}$

The last group of features considers the distances to the neighbouring points. To efficiently determine the nearest neighbours and the distances to them, the points are organized in a kd-tree. In contrast to the features describing the distribution of the point cloud, these features encode the relations between neighbouring points.

Let $\widetilde{d}_{i}$ be the distances to the nearest neighbour of $p_{i}$ and $\widetilde{D}$ the set of these distances:

$$
\widetilde{D}=\left\{\widetilde{d}_{1}, \widetilde{d}_{2}, \ldots, \widetilde{d}_{n}\right\}, \text { with } \widetilde{d}_{i}=\min _{i \neq j \in[1, n]} \operatorname{dist}\left(p_{i}, p_{j}\right)
$$

$\widetilde{D}$ is normalized using the maximum distance between two neighbours. Using the normalized set $\widetilde{d}_{i}$, the following nearest neighbours (NN) features are determined:

- Feature 71: Mean of the distances to the NN

- Feature 72: Median of the distances to the nearest NN

- Feature 73: Variance of the distances to the NN

- Feature 74: Standard dev. of the distances to the NN

- Feature 75-83: Histogram of the distances to the NN

\section{Classification}

Our approach to learn to classify the plants is based on supervised learning. For the learning process we generated a training set with known samples and trained the classifiers offline. The learning process is shown in Figure 2. For that we used the machine learning toolbox Weka [5], which supplies a large number of learning algorithms. Using the Weka explorer the learning methods can be easily and efficiently compared. Weka is Java based and outputs a model for each classifier as a Java class file, but also the classifier in text form as a function, tree, and so on, depending on the used learning method. Using this output, a classifier can be easily implemented. The classification process should run in realtime and is also shown in Figure 2.
Which classifier is best for our application is hard to predict, so we grouped them and compared the groups and the individual classifiers empirically. The chosen classifier groups and classifiers are listed in table II. The results of the comparison between the groups and the individual classifiers are shown in section IV.

\section{EXPERIMENTS}

The main objective of this work is to show, that it is possible to distinguish multiple plants using a low resolution 3D laser sensor and fast to calculate features. In this section the used 3D laser sensor is introduced, further we present the tested plants and describe how this dataset was recorded. This is followed by the experimental results, with the comparison of different classifiers and the evaluation of the used features.

\section{A. The FX6 3D laser sensor}

The FX6 sensor by Nippon Signal [14] is a lightweight 3D laser sensor. Is consists of no rotational mechanical parts and is thus robust against mechanical stress, but has a low resolution $(59 \times 29$ rays). It measures the distance and reflectance intensity using an infrared pulsed laser light with up to $16 \mathrm{fps}$, determining the time-of-flight at a smallest unit of about 30 picoseconds, which leads to a ranging precision of about $1 \mathrm{~cm}$. The laser beam is reflected by a mirror oscillating independently into two directions. The combination of these oscillations results in a Lissajous figure. The main advantage of the FX6 sensor is its reliability under changing lighting conditions, e.g., the sensor is not influenced by the sunlight and by other weather conditions. Using a FX6 sensor it is even possible to operate 24 hours a day and handle conditions like light fog and dust. In agricultural environments such conditions may occur. A more detailed description and comparison with other 3D technologies, like PMD cameras and stereo vision, can be found in [4].

\section{B. Tested plants}

To generate a training set, we decided to use potted plants available in common nurseries, and sensed them separately from each other in the laboratory. This relieved the collection of the data, and we got a good and clean database. For the database we have chosen the plants shown in Figure 5. In this figure an example image of each chosen plant species and the corresponding point cloud is displayed.

To distinguish plant species it is not sufficient to use one example per species, otherwise we would learn this particular example plant. Due to that, we have used plants from nurseries where greater quantities of one species are available. In Table I the number of samples and the number of datasets per species are stated. To generate the datasets we have used different adjustments of the laser sensor and also rotated the plants, to get different views of each plant. Besides the plants, we also sensed the ground to be able to 

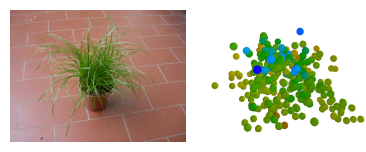

(a) Cyperus Zumula

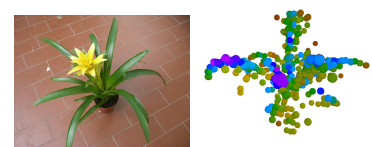

(c) Guzmania Bromelia
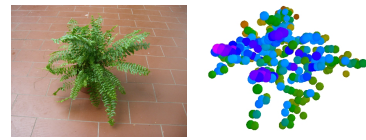

(e) Nephrolepis Exaltata
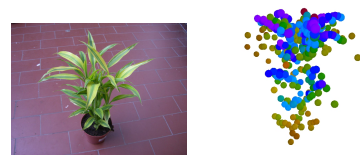

(b) Dracaena Sanderina
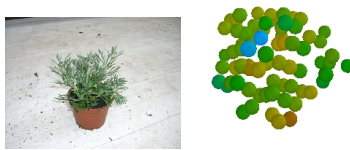

(d) Leucanthemum $\mathrm{H}$.
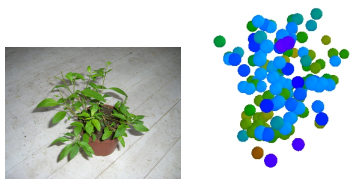

(f) Solanum Jasminoides
Figure 5. The images (a)-(f) show our six tested plants species.

Table I

NUMBER OF SAMPLES AND DATASET PER PLANT SPECIES

\begin{tabular}{|r|c|c|}
\hline Plant species & $\begin{array}{c}\text { Number of } \\
\text { samples }\end{array}$ & $\begin{array}{c}\text { Number of } \\
\text { datasets }\end{array}$ \\
\hline Cyperus Zumula & 20 & 140 \\
Dracaena Sanderina & 8 & 100 \\
Guzmania Bromelia & 20 & 158 \\
Leucanthemum Hosmariense & 23 & 100 \\
Nephrolepis Exaltata & 12 & 127 \\
Solanum Jasminoides & 24 & 100 \\
\hline
\end{tabular}

determine the plant coordinate system, which is essential for the feature calculation.

\section{Classifier evaluation}

In our first experiments we have compared the classifiers listed in section III-D using the Weka explorer and all of the 83 features. To evaluate the performance of these classifiers we ran a ten-fold cross-validation ten times and took the mean of the percentage of correctly classified instances for each classifier. Table II shows these results sorted by the classifier groups. The classifiers of each group are sorted according to the performances.

The evaluation has shown, that the classification performance strongly depends on the classifier used. The best classifiers achieved above $98 \%$ of correctly classified instances, the worst just $34.46 \%$. Each group has a classifier with a detection rate above $87 \%$, but the functions group outperforms the others. Each of these classifiers achieved a detection rate above $92 \%$.

The best classifiers were LMT [15], a classifier for building logistic model trees, which are classification trees with logistic regression functions at the leaves, SimpleLogistic [15], a classifier for building linear logistic regression models, SMO [16][17] an implementation of John Platt's
Table II

CLASSIFIER EVALUATION

\begin{tabular}{|c|c|c|}
\hline $\begin{array}{r}\text { Classifier } \\
\text { Group }\end{array}$ & Classifier & $\begin{array}{l}\text { Avg. Correctly } \\
\text { Classified [\%] }\end{array}$ \\
\hline Bayes & BayesNet & 89.62 \\
\hline \multirow[t]{4}{*}{ classifier } & NaiveBayes & 87.01 \\
\hline & NaiveBayesUpdateable & 87.01 \\
\hline & NaiveBayesMultinomial & 46.18 \\
\hline & ComplementNaiveBayes & 34.46 \\
\hline \multirow[t]{7}{*}{ Trees } & LMT & 98.8 \\
\hline & RandomForest & 92.97 \\
\hline & $\mathrm{J} 48$ & 85.82 \\
\hline & NBTree & 85.68 \\
\hline & REPTree & 82.3 \\
\hline & RandomTree & 69.08 \\
\hline & DecisionStump & 37.85 \\
\hline \multirow[t]{6}{*}{ Rules } & Nnge & 89.53 \\
\hline & PART & 86.76 \\
\hline & Jrip & 85.06 \\
\hline & Ridor & 84.8 \\
\hline & DecisionTable & 74.76 \\
\hline & ConjunctiveRule & 38.21 \\
\hline \multirow[t]{5}{*}{ Functions } & SimpleLogistic & 98.8 \\
\hline & SMO & 98.37 \\
\hline & MultilayerPerceptron & 98.14 \\
\hline & RBFNetwork & 94.55 \\
\hline & Logistic & 92.46 \\
\hline \multirow{4}{*}{$\begin{array}{r}\text { Lazy } \\
\text { classifier }\end{array}$} & IB1 & 92.04 \\
\hline & Ibk & 92.04 \\
\hline & Kstar & 90.14 \\
\hline & LWL & 49.25 \\
\hline \multirow[t]{2}{*}{ Misc. } & HyperPipes & 87.84 \\
\hline & VFI & 84.79 \\
\hline
\end{tabular}

sequential minimal optimization algorithm for training a support vector classifier, which solves the multi-class problems using pairwise classification, and MultilayerPerceptron a neuronal network which uses backpropagation to classify instances. The two best classifiers, LMT and SimpleLogistic, which achieved a detection rate of about $99 \%$, are closely related. The tree build by LMT uses SimpleLogistic at the leaves, but in our case it turned out that the tree reduces to one node.

Using the presented feature set the classification runs in real-time for all classifiers. The time consuming task is the feature extraction, but runs also in real-time. What takes time is the training phase. Using the 725 instances in the database, the training phase of SMO took less than one second, of SimpleLogistic about 10 seconds, of LMT 30 seconds and of MultilayerPerceptron about a minute in average. The high detection rates and short computational time have shown that the proposed method is sufficient for a reliable classification of plant species in real-time.

\section{Feature evaluation}

We have used the entire set of 83 features to evaluate the classifiers. To see which characteristics are particularly important for the classification, these features were divided into six groups as listed in table III. To compare these groups we have used the above mentioned four methods, which 
Table III

FEATURE EVALUATION

\begin{tabular}{|r|l|l|l|l|}
\hline \multirow{2}{*}{ Feature Group } & \multicolumn{3}{|c|}{ Avg. Correctly Classified Plants [\%] using } \\
& LMT & $\begin{array}{l}\text { Simple } \\
\text { Logistic }\end{array}$ & SMO & $\begin{array}{l}\text { Multilayer } \\
\text { Perceptron }\end{array}$ \\
\hline Entire Features Set (1-83) & 98.8 & 98.8 & 98.14 & 98.37 \\
\hline Reflectance Based (1-16) & 85.93 & 85.93 & 81.25 & 85.24 \\
\hline 3D Point Cloud Distribution (17-31) & 81.38 & 77.66 & 72.14 & 82.76 \\
\hline 2D Projection on y-z-Plane (32-44) & 61.24 & 54.21 & 51.45 & 57.93 \\
\hline 2D Projection on x-z-Plane (45-57) & 70.48 & 70.62 & 69.66 & 71.45 \\
\hline 2D Projection on x-y-Plane (58-70) & 50.07 & 49.93 & 51.03 & 50.46 \\
\hline 3D Neighbourhood (71-83) & 61.66 & 60.14 & 52.41 & 57.93 \\
\hline
\end{tabular}

yielded the best results when using all the features, and trained the classifiers again with each group. The comparison shows that the most important features are the reflectance based and the 3D point cloud distribution features. Each of these groups yielded a detection rate of over $80 \%$ when used alone.

\section{CONCLUSiOnS}

In this paper we have introduced a method for distinguishing plant species by using a low resolution 3D LIDAR sensor and supervised learning. For this purpose we have developed an easy to compute feature set with common statistical features which are independent of the plant size. We have trained and compared several classifiers with this feature set. It turned out that the best methods for our application are simple logistic regression functions, support vector machines and neuronal networks. In the laboratory we used plants of six different species and were able to classify nearly $99 \%$ of these plants correctly.

\section{REFERENCES}

[1] B. J. Legg and J. V. Stafford, "Precision agriculture - new technologies," in Proceedings of The Brighton Conference Pest and Diseases, 1998, pp. 1143-1150.

[2] Y. Edan, S. Han, and N. Kondo, "Automation in agriculture," in Handbook of Automation. Springer, 2009, pp. 1095-1128.

[3] A. Ruckelshausen, P. Biber, M. Dorna, H. Gremmes, R. Klose, A. Linz, F. Rahe, R. Resch, M. Thiel, D. Trautz, and U. Weiss, "Bonirob - an autonomous field robot platform for individual plant phenotyping," in Proceedings of Joint International Agricultural Conference, 2009, p. 464pp.

[4] U. Weiss and P. Biber, "Plant detection and mapping for agricultural robots using a 3d-lidar sensor," in Proceedings of the 4th European Conference on Mobile Robots, 2009, pp. 205-210.

[5] I. H. Witten and E. Frank, Data Mining: Practical Machine Learning Tools and Techniques. Kaufmann, M., 2005, vol. 2.

[6] L. Tang and J. Jin, "Corn plant sensing with active and passive stereo vision," International Conference on Robotics and Automation, vol. 1, pp. 31-37, 2008.
[7] A. Paap, S. Askraba, K. Alameh, and J. Rowe, "Photonicbased spectral reflectance sensor for ground-based plant detection and weed discrimination," Optics Express, vol. 16, no. 2, pp. 1051-1055, 2008.

[8] Y. Yovel, M. O. Franz, P. Stilz, and H.-U. Schnitzler, "Plant classification from bat-like echolocation signals," PLOS Computational Biology, vol. 4, pp. 1-13, 2008.

[9] M. Beigi, M. Wang, and A. Zell, "Time-resolved spectrum kernel for biosonar target classification," in Proceedings of the 4th Conference on Signal Processing, Pattern Recognition, and Applications, 2007.

[10] M. Wang and A. Zell, "Sequential sensing with biosonar for natural landmark classification," in Proceedings of IEEE International Safety, Security and Rescue Robotics, Workshop, 2005, pp. 137-142.

[11] S. Wu, F. Bao, E. Xu, Y.-X. Wang, Y.-F. Chang, and Q.-L. Xiang, "A leaf recognition algorithm for plant classification using probabilistic neural network," in IEEE International Symposium on Signal Processing and Information Technology, 2007, pp. $11-16$.

[12] J. Böhm and C. Brenner, "Curvature based range image classification for object recognition," in Proceedings of Intelligent Robots and Computer Vision XIX: Algorithms, Techniques, and Active Vision, 2000, pp. 211-220.

[13] G. Hetzel, B. Leibe, P. Levi, and B. Schiele, "3d object recognition from range images using local feature histograms," in Proceedings of Conference on Computer Vision and Pattern Recognition, 2001, pp. 394-399.

[14] Nippon-Signal, "FX6," http://www.signal.co.jp/, January 2009.

[15] N. Landwehr, M. Hall, and E. Frank, "Logistic model trees," Machine Learning, vol. 59, no. 1-2, pp. 161205, 2005. [Online]. Available: http://dx.doi.org/10.1007/ s10994-005-0466-3

[16] J. C. Platt, "Fast training of support vector machines using sequential minimal optimization," in Proceedings of Advances in kernel methods: support vector learning, 1998, pp. 185208 .

[17] S. S. Keerthi, S. K. Shevade, C. Bhattacharyya, and K. R. K Murthy, "Improvements to platt's smo algorithm for svm classifier design," Neural Computation, vol. 13, no. 3, pp. 637-649, 2001. 